\title{
ANÁLISE DA APLICAÇÃO DO SEIS SIGMA EM UM SETOR DA INDÚSTRIA AUTOMOTIVA: UM ESTUDO DE CASO
}

\author{
Henrique Monte Mor Silva ${ }^{1}$ e Felipe Araújo Calarge ${ }^{2}$ \\ Universidade Nove de Julho \\ E-mails: $\underline{\text { hmontemor@gmail.com, fcalarge@ uninove.br }}$
}

\section{RESUMO}

A busca por vantagens competitivas cria a necessidade das organizações absorverem técnicas que aumentem a viabilidade técnico econômica de seus processos e produtos para uma maior rentabilidade. Uma abordagem que vem sendo aplicada para redução de variabilidade e custos envolvidos em processos é o Seis Sigma, o qual é suportado por técnicas de gestão da qualidade com enfoque estatístico. Este trabalho buscou analisar a aplicação do Programa Seis Sigma de forma descritiva e detalhada, uma vez que são escassos os trabalhos neste campo de aplicação. A presente pesquisa é, portanto, de natureza aplicada com objetivo exploratório e usou como método o estudo de caso e, como instrumento de coleta de dados, a entrevista semiestruturada. $\mathrm{O}$ estudo de caso foi realizado em um setor da indústria automotiva, onde foi desenvolvido um projeto Seis Sigma que envolveu a substituição da matéria prima usada na fabricação de componentes de limpadores de para-brisas. Por meio da análise dos resultados, verificou-se uma redução de gastos com matéria prima, melhoria de confiabilidade do processo e aumento da satisfação do cliente. Dessa forma, existem evidências de que o Seis Sigma proporciona benefícios consideráveis à organização, entretanto, sua implementação deve ser rigorosamente estruturada.

\section{ANALYSIS OF SIC SIGMA APPLICATION IN NA AUTOMOTIVE INDUSTRY SECTOR: A CASE STUDY}

\begin{abstract}
The search for competitive advantages creates the need for organizations to absorb techniques that increase the technical economic viability of their processes and products to a greater profitability . One approach that has been applied to reduce variability and costs involved in processes is the Six Sigma, which is supported by quality management techniques with statistical approach . This study aimed to analyze the application of the Six Sigma Program in a descriptive and detailed way, since there are few studies in this application field. Therefore, this is an applied research with exploratory goal which used the case study method and the semi-structured interview as the data collection instrument. The case study was conducted in a sector of the automotive industry, in which it has been developed a Six Sigma project that involved a replacement of the raw material used in the manufacture of windshield wiper components. Through the result analysis, it was possible to verify a reduction of spending on raw materials, an improvement in process reliability and customer satisfaction increase. Thus,
\end{abstract}


there are evidences that Six Sigma provides substantial benefits to the organization, however, its implementation must be carefully structured .

\section{INTRODUÇÃO}

Em virtude da alta competitividade, as organizações buscam meios de viabilizar seus produtos e serviços por meio da melhoria contínua em seus processos. Isto visa a redução de desperdícios, da variabilidade e, primordialmente, dos custos, para que haja um aumento da produtividade e do lucro. Existem diversas práticas e técnicas pelas quais se podem alcançar tais objetivos (RAMOS et al., 2013 [1]). Uma prática que tem sido explorada por diversas organizações é o Seis Sigma, uma técnica que reúne poderosos métodos estatísticos e científicos para diminuição de defeitos com foco nas necessidades dos clientes (MAST e LOKKERBOL, 2012 [2]).

O Seis Sigma utiliza um método chamado DMAIC. Esse método é conhecido como um meio de redução da variabilidade de processos, mas, na prática, pode ser aplicado como uma técnica de resolução de problemas (MAST; LOKKERBOL, 2012 [2]). O DMAIC é, portanto, uma ferramenta na implementação do Seis Sigma e responsabiliza-se pela melhoria do processo, podendo ser ainda abrangido no campo da engenharia da qualidade, abordando ideias de controle estatístico e gerenciamento da qualidade total (MAST; LOKKERBOL, 2012 [2]). A implementação do Seis sigma e seu método DMAIC engloba várias ferramentas para análise e melhoria do processo, como por exemplo, as cartas de controle, os histogramas e os testes de hipóteses paramétricos (SATOLO et al., 2009 [3]).

Dada a relevância da técnica, este trabalho procurou demonstrar sua aplicação em um projeto de redução de custos em relação a produtos provenientes de um processo de estampagem em um setor da indústria automotiva, em cuja empresa analisada, a maioria dos produtos estampados eram fabricados utilizando-se como matéria prima o aço relaminado com baixo teor de carbono (ABNT NBR 5007 [4]) e o aço laminado a frio (ABNT NBR 5915-2 [5]).

Nas próximas seções serão apresentados o referencial teórico sobre o Seis Sigma, o método de pesquisa no qual a pesquisa foi fundamentada, o estudo de caso demonstrando cada fase do DMAIC, as análises dos resultados obtidos e as conclusões às quais se pôde chegar.

\section{REFERENCIAL TEÓRICO}

O Seis Sigma tem a finalidade de aumentar a capacidade dos processos de uma organização, por meio de ferramentas adequadas, a fim de melhorar o desempenho e diminuir a variabilidade (YANG; El-HAIK, 2008 [6]). Trata-se, portanto, de uma abordagem que melhora o desempenho de todo o negócio e atua na satisfação dos clientes, apoiando-se na aplicação de técnicas estatísticas e na utilização de indicadores de desempenho para a avaliação e otimização de processos, proporcionando ainda, válida aprendizagem advinda do comprometimento e da capacitação de pessoal (SANTOS; MARTINS, 2010 [7]).

De maneira geral, o Seis Sigma possibilita o alcance do sucesso na competitividade através da melhoria contínua dos processos de produção de maneira a envolver todos os aspectos estratégicos, sucesso esse que só pode ser alcançado por meio da aplicação de determinadas 
técnicas e ferramentas que ajudam a definir os problemas a serem atacados, visando o maior retorno financeiro possível (SATOLO et al., 2009 [3]). Essas técnicas também proporcionam a obtenção de dados do processo para uma análise crítica, por meio da qual são definidas as atividades necessárias para a melhoria e, finalmente, proporcionam a implementação de procedimentos de controle para garantir a permanência das melhorias alcançadas (SATOLO et al., 2009 [3]).Trabalhar em um padrão Seis Sigma significa gerar 3,4 unidades produzidas com defeito em cada milhão de oportunidades, o que implica baixa variabilidade no processo (YANG e El-HAIK, 2008 [6]).

O Seis Sigma utiliza duas principais ferramentas: o DMAIC e o DFSS. O DMAIC é comumente aplicado à redução da variabilidade em processos, mas, na prática, existe a possibilidade de aplicá-lo à resolução de problemas (MAST; LOKKERBOL, 2012 [2]). As fases do DMAIC referem-se à sigla e são: Define (definir), Measure (medir), Analyze (analisar), Improve (melhorar) e Control (controlar) (SHAFER; MOELLER, 2012 [8]). Já o DFSS (Design for Six Sigma) é uma ferramenta usualmente aplicada a processos novos e, conincidentemente, é também dividido em cinco fases (DMADV): Define (definir), Measure (medir), Analyze (analisar), Design (projetar) e Verify (verificar) (BANUELAS; ANTONY, 2003 [9]).

Embora o Seis Sigma seja bem estruturado e proporcione muitos benefícios, principalmente econômicos, sua implementação não se dá de forma simples, pois alguns fatores dentro das organizações podem inibir seu uso, sendo um dos fatores mais relevantes o envolvimento da alta administração. (CHAKRAVORTY, 2009 [10]). Além disso, em uma survey feita por Carvalho, Ho e Pinto (2007) [11], de 198 empresas brasileiras que responderam a um questionário sobre implementação do Seis Sigma, somente 46 faziam uso da técnica. Isso gera a necessidade de que a metodologia seja maiormente disseminada nas organizações.

\section{MÉTODO DE PESQUISA}

Esta é uma pesquisa qualitativa e exploratória (MARCONI; LAKATOS, 2010 [12]), uma vez que se procurou, primordialmente, demonstrar a utilização do Seis Sigma e seu método DMAIC em um setor da indústria automotiva, sem que houvesse uma variável de medida específica para a determinação da eficiência da técnica. Pelo contrário, buscou-se demonstrála e explorá-la detalhadamente. Quanto ao método, escolheu-se o estudo de caso e, por se tratar de um estudo exploratório, um único caso foi considerado suficiente (YIN, 2010 [13]). A coleta de dados foi feita por meio de entrevista semiestruturada.

A escolha do setor e da organização para a análise foi feita sob conveniência dos pesquisadores. Dessa forma as análises puderam contar com um maior nível de aprofundamento a fim de que os resultados tivessem o maior índice de confiabilidade possível. A empresa analisada é de grande porte, brasileira e líder no seu segmento. O estudo de caso foi feito sobre um projeto Seis Sigma que teve como objetivo a redução de custos relacionados aos produtos fabricados em aço relaminado e laminado em um processo de estampagem. As entrevistas foram feitas com os gerentes de engenharia de produto, da engenharia industrial e da área comercial, além dos green belts e dos envolvidos na equipe do projeto Seis Sigma estudado. Em acordo com os procedimentos da empresa, foram disponibilizados dados suficientes pelos quais foi possível fazer uma análise com alto nível de detalhes, explorando cada fase do DMAIC. 


\section{ESTUDO DE CASO - DEFINIÇÃO DO PROBLEMA E DAS METAS}

Na primeira fase do DMAIC, etapa definir (D), percebeu-se pelas análises de retorno financeiro que, dentre diversas oportunidades para desenvolvimento do projeto Seis Sigma, no momento, o mais vantajoso seria voltar todos os esforços para a redução de custos no uso dos aços relaminados, que tinha significativa representatividade nos gastos anuais com produção, com pouco mais de $\mathrm{R} \$ 3.716 .104,80$, o que corresponde a aproximadamente $3 \%$ do faturamento anual. A meta definida foi uma redução de $20 \%$ desse valor. Na empresa analisada, esse material é comprado em 19 variações, em relação às suas dimensões. Os gastos anuais com cada variação estão representados em um gráfico de Pareto na figura 1. Percebe-se que as variações de P1 a P7 representam $80 \%$ dos gastos com o material.

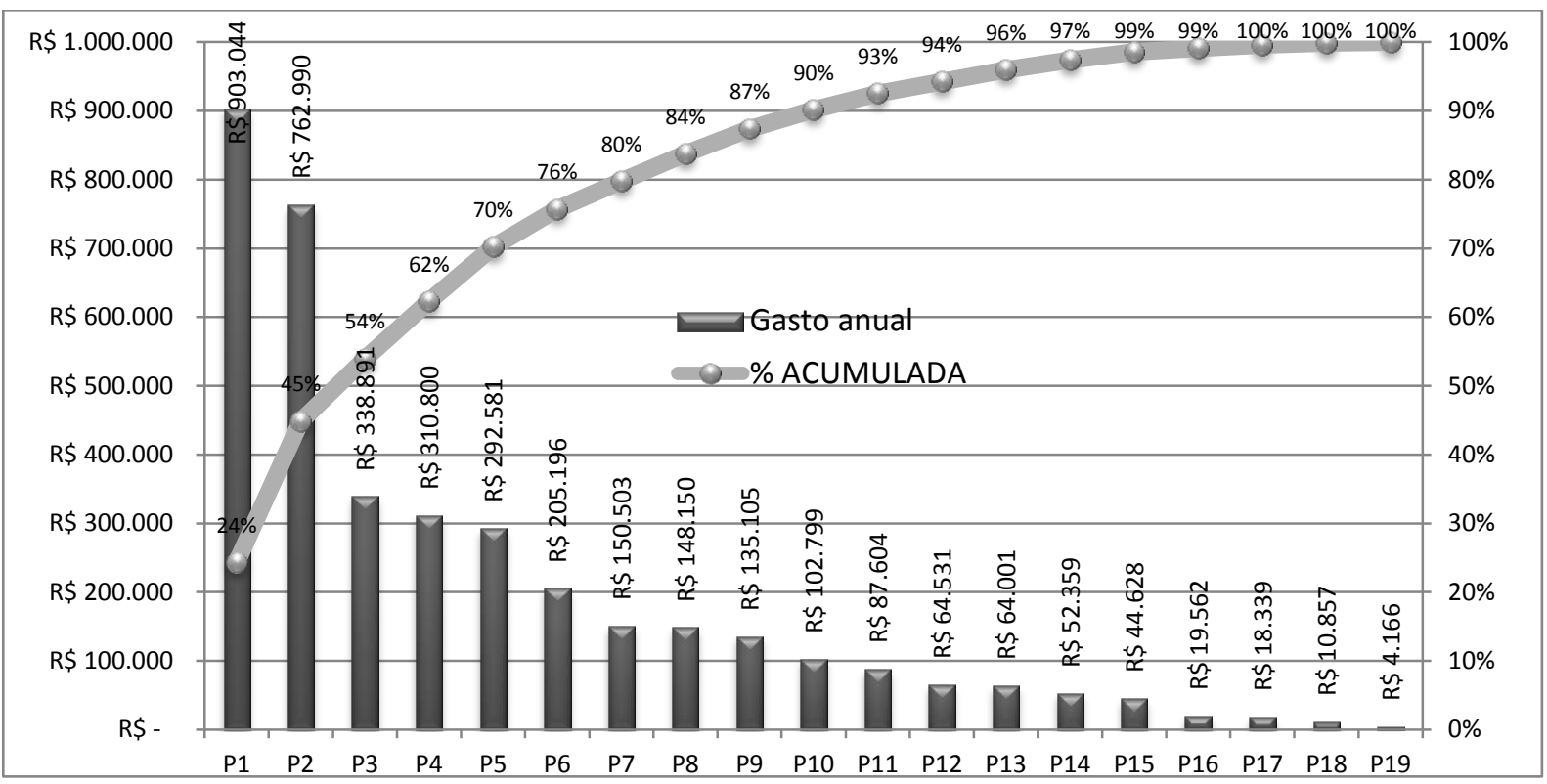

FIGURA 1 - Pareto - Gastos anuais com compra de material relaminado

Os processos de produção dos produtos que usam esse material, nas suas 19 variações, estavam em condições de variabilidade aceitáveis, segundo os padrões impostos pelos clientes. Isso inibia as intenções de melhoria no processo, especificamente. Dessa forma, após um brainstorming, constatou-se que a melhor opção cabível seria uma possível substituição da materia prima. Eram usados apenas dois tipos de materiais: o aço carbono laminado conforme a ABNT NBR 5915-2 [5] e o aço carbono relaminado conforme ABNT NBR 5007 [4]. Obviamente, o aço relaminado custa mais caro do que o laminado. Logo, decidiu-se avaliar a possibilidade de substituir a materia prima dos produtos que usavam o aço relaminado por aço laminado.

\section{MEDIÇÃO}

Passou-se então para a fase medir (M) do DMAIC, onde foram feitas algumas medições para posterior comparação entre os dois materiais. Para tanto, foi escolhido o produto mais crítico, tanto em termos de produtividade quanto em termos de desempenho funcional. Esse produto faz parte de um braço de limpador de para-brisas e é denominado dobradiça. A figura 20 ilustra. 


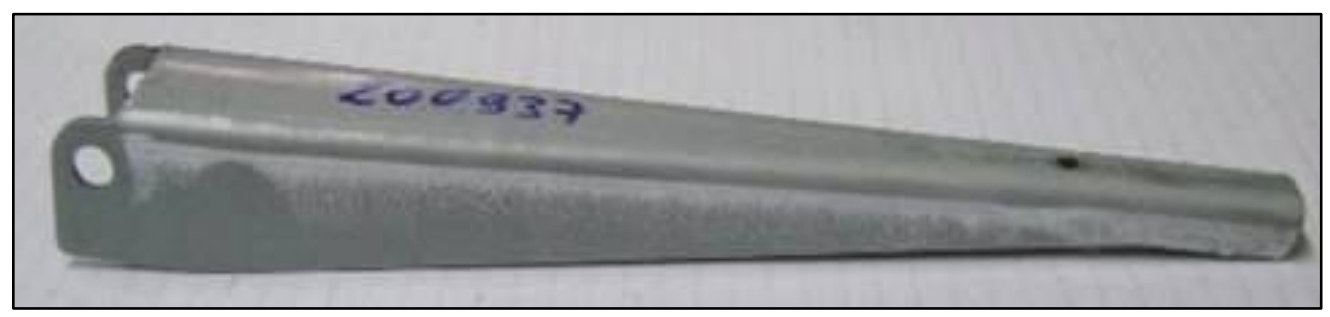

FIGURA 2 - Dobradiça

A primeira medição visou a variabilidade no processo de estampagem. O produto escolhido tem 5 medidas que são usadas para controle do processo. No entanto, por questões de tempo e custo, foi escolhida apenas a mais crítica, que, por se tratar de uma peça cuja secção transversal tem formato de "U", é a largura interna. Assim, a análise foi feita por meio de gráficos de controle e histogramas. Para tanto, foram coletadas amostras suficientemente grandes (MONTGOMERY, 2004 [14]) e os gráficos de controle e histogramas foram gerados com o auxilio do software Minitab 16. As figuras 3 e 4 mostram os resultados.

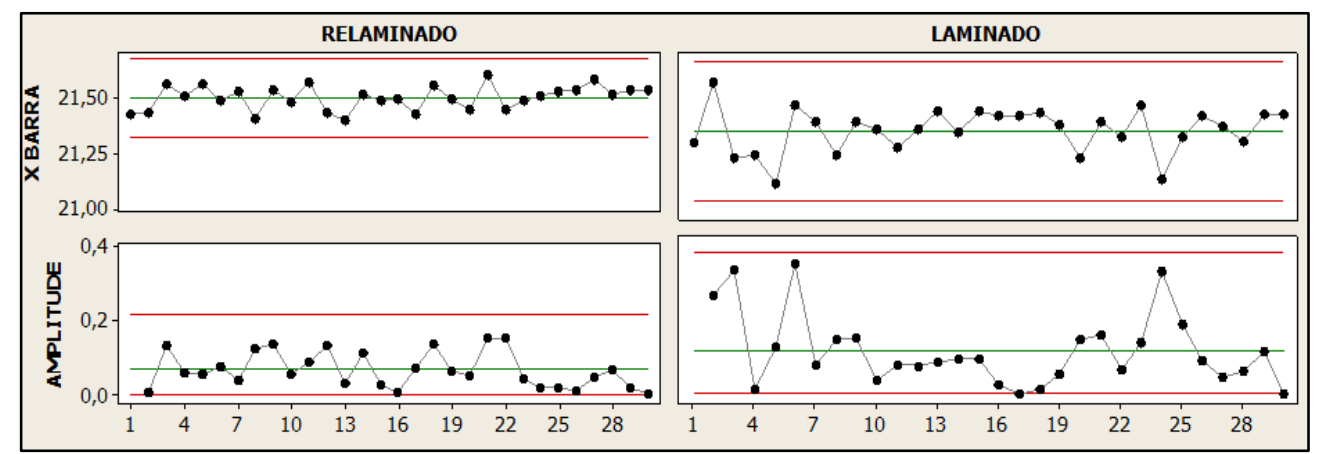

FIGURA 3 - Estratificação dos dados - Cartas de controle

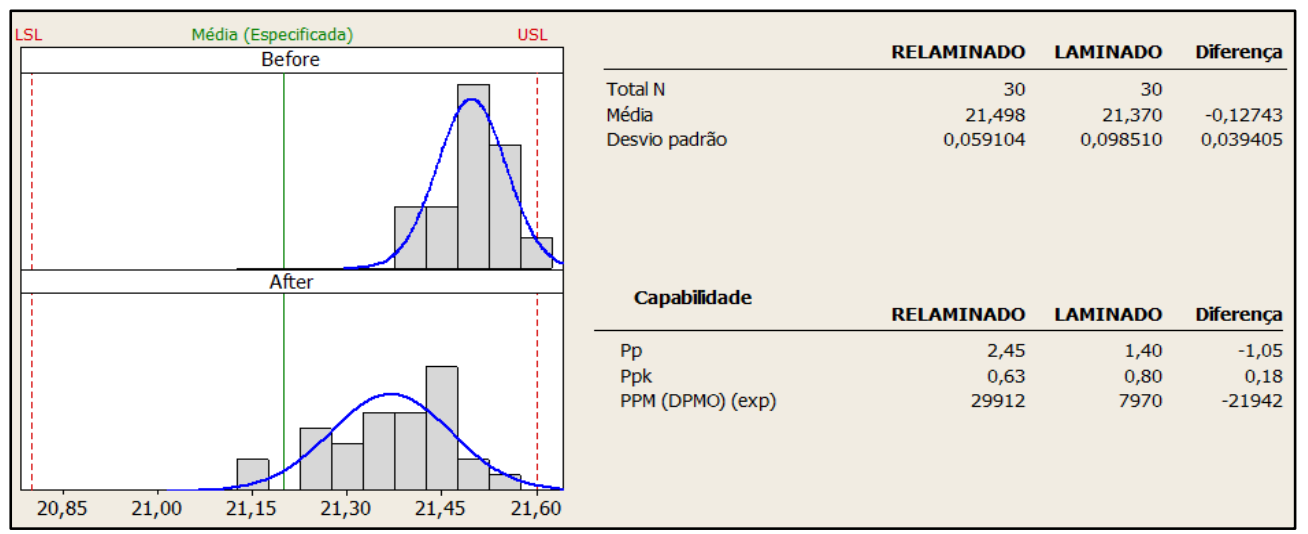

FIGURA 4 - Estratificação dos dados - Histogramas

Através das cartas de controle e dos histogramas gerados, percebeu-se que o processo com o material relaminado, apesar de ser considerado aceitável pela empresa, já apresentava dificuldades em relação ao controle, visto pelo ppk de 0,63. Entretanto, a variabilidade estava bastante adequada, com pp de 2,45. Em comparação com o material laminado, pelos gráficos e valores, pode-se dizer que houve redução do pp e aumento do ppk. Consequentemente, nota-se que as epectativas de DPMO diminuiram, de 29912 para 7970. Numa análise superficial, também pode-se dizer que a média reduziu e o desvio padrão aumentou, de 
$21,498 \mathrm{~mm}$ para $21,370 \mathrm{~mm}$ e de $0,059 \mathrm{~mm}$ para $0,098 \mathrm{~mm}$, respectivamente. Entretanto, foram necessárias análises estatísticas mais profundas para confirmação das mudanças, o que foi feito na etapa seguinte do DMAIC, fase analisar.

Na seqüência, ainda na fase medir, fez-se uma comparação em relação à funcionalidade do produto. Esse produto é submetido a intensos e repetitivos esforços, quando em funcionamento, e demanda um maior tempo de durabilidade em relação aos demais produtos que compões o braço de um limpador de pára brisas. Segundo à norma de produto interna da empresa, para garantir um bom funcionamento nas condições descritas, esse produto deve ser submetido a um ensaio chamado carga de neve. Como explicado anteriormente, é um produto constituinte de um sistema de limpador de pára brisas (SLPB). Fica criticamente engastado entre dois outros componentes.

O ensaio de carga de neve, segundo informações dos engenheiros de produto da empresa analisada, consiste em avaliar o funcionamento do SLPB em condições climáticas de baixa temperatura, em que há a possibilidade de acúmulos de neve no pára brisas, dificultando o movimento cíclico completo do SLPB. Isso força o SLPB, fazendo com que seu desgaste seja acelerado, sendo que, dentre os produtos que compõem o SLPB, o produto referido é o de maior criticidade nessas condições de funcionamento. A durção do ensaio é de 500.000 ciclos. Assim, foram submetidos ao ensaio quatro conjuntos de SLPB: dois com produtos de aço relaminado e dois com produtos de aço laminado. Todos os conjuntos apresentaram-se em condições aceitáveis de uso ao final dos 500.000. Isso significa que o produto, tanto fabricado em aço relaminado quanto em aço laminado, atende às severas condições de funcionamento estabelecidas pelas normas da empresa.

Posteriormente, passou-se para a terceira e última medição da fase medir do DMAIC. Essa medição consistiu em avaliar a resistência à desconexão entre o produto analisado e os componentes adjacentes. O produto analisado passa por uma etapa de cravação no processo de montagem do SLPB. Segundo às normas de produto internas da empresa, essa cravação deve ser avaliada submetendo-se o produto a um ensaio de arrancamento por tração, que visa medir a força para desconexão dos componentes. Segundo as especificações de tais normas, essa força deve ser superior a 3800N. Dessa forma, foram avaliadas 30 amostras do produto em nálise para cada cada tipo de material. Os valores encontrados são apresentados na tabela 1.

TABELA 1 - Valores encontrados no esnsaio de arrancamento por tração

\begin{tabular}{|c|c|c|c|c|c|c|c|c|}
\hline AMOSTRA & RELAMINADO & LAMINADO & AMOSTRA & RELAMINADO & LAMINADO & AMOSTRA & RELAMINADO & LAMINADO \\
\hline 1 & $5260,5 \mathrm{~N}$ & $4499,4 \mathrm{~N}$ & 11 & $5501,6 \mathrm{~N}$ & $4886,1 \mathrm{~N}$ & 21 & $4973,5 \mathrm{~N}$ & $4850,3 \mathrm{~N}$ \\
\hline 2 & $5176,6 \mathrm{~N}$ & $4880,2 \mathrm{~N}$ & 12 & $5437,7 \mathrm{~N}$ & $4522,5 \mathrm{~N}$ & 22 & $5083,6 \mathrm{~N}$ & $5013,8 \mathrm{~N}$ \\
\hline 3 & $5003,8 \mathrm{~N}$ & $4403,2 \mathrm{~N}$ & 13 & $4996,1 \mathrm{~N}$ & $4831,1 \mathrm{~N}$ & 23 & $5436,5 \mathrm{~N}$ & $4684,5 \mathrm{~N}$ \\
\hline 4 & $5249,5 \mathrm{~N}$ & $4804,4 \mathrm{~N}$ & 14 & $4961,4 \mathrm{~N}$ & $4924,6 \mathrm{~N}$ & 24 & $5386,4 \mathrm{~N}$ & $4774,1 \mathrm{~N}$ \\
\hline 5 & $5190,7 \mathrm{~N}$ & $4776,4 \mathrm{~N}$ & 15 & $5431,7 \mathrm{~N}$ & $4931,2 \mathrm{~N}$ & 25 & $5016,7 \mathrm{~N}$ & $5175,6 \mathrm{~N}$ \\
\hline 6 & $5359,9 \mathrm{~N}$ & $4751,6 \mathrm{~N}$ & 16 & $4974,5 \mathrm{~N}$ & $5016,9 \mathrm{~N}$ & 26 & $4871,6 \mathrm{~N}$ & $4879,6 \mathrm{~N}$ \\
\hline 7 & $4472,7 \mathrm{~N}$ & $4802,5 \mathrm{~N}$ & 17 & $5022,2 \mathrm{~N}$ & $5053,7 \mathrm{~N}$ & 27 & $4837,9 \mathrm{~N}$ & $5005,7 \mathrm{~N}$ \\
\hline 8 & $4874,6 \mathrm{~N}$ & $5046,1 \mathrm{~N}$ & 18 & $4978,9 \mathrm{~N}$ & $4716,8 \mathrm{~N}$ & 28 & $4768,5 \mathrm{~N}$ & $5537,4 \mathrm{~N}$ \\
\hline 9 & $5012,9 \mathrm{~N}$ & $4791,5 \mathrm{~N}$ & 19 & $5510,3 \mathrm{~N}$ & $4170,6 \mathrm{~N}$ & 29 & $5094,7 \mathrm{~N}$ & $4671,1 \mathrm{~N}$ \\
\hline 10 & $5214,6 \mathrm{~N}$ & $4890,7 \mathrm{~N}$ & 20 & $4797,1 \mathrm{~N}$ & $4889,5 \mathrm{~N}$ & 30 & $5228,0 \mathrm{~N}$ & $5335,9 \mathrm{~N}$ \\
\hline
\end{tabular}


Pela tabela dos valores encontrados no ensaio de arrancamento por tração, extrai-se que, para o produto fabricado com material relaminado, a força média obtida foi de $5104,2 \mathrm{~N}$, com desvio padrão de $247,8 \mathrm{~N}$ e força mínima encontrada de $4472,7 \mathrm{~N}$. Já para o produto fabricado com o material laminado, obteve-se uma força média de $4850,6 \mathrm{~N}$, com desvio padrão de 261,9N e força mínima de 4170,6N. A julgar apenas por essa análise superficial, pode-se afirmar que a força média diminuiu e o desvio padrão aumentou, na substituição do material relaminado pelo laminado. Entretanto, foram realisadas análises estatísticas mais profundas para uma afirmação mais concreta.

\section{ANÁLISE DOS DADOS, MELHORIA E CONTROLE}

Relizadas as medições, proseguiu-se para a fase analisar (A) do DMAIC. Nesta fase, o propósito foi verificar, por meio de técnicas estatísticas, se as afirmações previamente feitas em relação aos resultados obtidos eram de fato verdadeiras. Em relação à segunda medição, que visou a avaliar a durabilidade do produto, não houve a necessidade de maiores análises, uma vez que o objetivo era que o produto resistisse a 500.000 ciclos de funcionamento sem interrupção e permanecesse em boas condições de uso. Como as amostras, tanto fabricadas com o material relaminado quanto fabricadas com o material laminado, passaram no teste, pode-se concluir que a mudança de material não influenciou no resultado desse ensaio. Já para as análises das medições referentes à variabilidade no processo de estampagem e à força de resistência ao arrancamento, foram realisadas maiores investigações com a aplicação de técnicas estatísticas avançadas, mais especificamente, os testes de hipóteses paramétricos para a diferença de médias e variâncias de populações com distribuições normais (RAMOS et al., $2013[1])$.

Quanto à variabilidade do processo, os resultados mostraram que, em relação à medida escolhida para a comparação entre os materiais, do relaminado para o laminado, a média reduziu de $21,498 \mathrm{~mm}$ para $21,370 \mathrm{~mm}$, e o desvio padrão aumentou de $0,059 \mathrm{~mm}$ para $0,098 \mathrm{~mm}$. A primeira análise para constatação da ocorrência visou verificar se de fato houve influência do material a ponto de gerar diferenças em relação ao desvio padrão. Assim, fez-se um teste $\mathrm{F}$ para inferir sobre a variância (desvio padrão ao quadrado), considerando-se $95 \%$ de confiança (MONTGOMERY, 2004 [14]):

Teste de hipótese

\section{Valor do Fcrítico}

$\mathrm{H} 0: s_{1}^{2}=s_{2}^{2}$

$$
F i=F_{29 ; 29 ; 5 \%}=0,53
$$

$\mathrm{H} 1: s_{1}^{2}<s_{2}^{2}$

sendo $s_{1}^{2}$ a variância para o material relaminado e $s_{2}^{2}$ a variância para o material laminado.

Estatística de teste:

$F_{\text {teste }}=\frac{\min \left(0,098^{2} ; 0,059^{2}\right)}{\max \left(0,098^{2} ; 0,059^{2}\right)}=\frac{0,059^{2}}{0,098^{2}}=0,36$

Como se observa, o Fteste $(0,36)$ apresentou-se menor do que o Fcrítico inferior $(0,53)$. Dessa forma, rejeitou-se $\mathrm{H} 0$ e aceitou-se $\mathrm{H} 1$. Isso significa que pode-se afirmar com $95 \%$ de confiança que a variância e, consequentemente, o desvio padrão aumentaram, do material relaminado para o laminado. 
Sabendo-se que as variâncias são diferentes, o teste mais adequado utilizado para inferir sobre as médias foi o teste $t$ para diferença de médias de duas populações com distribuições normais presumindo variâncias diferentes (MONTGOMERY, 2004 [14]).

Consideraram-se, portanto, as seguintes hipóteses:

H0: $\mu_{1}-\mu_{2}=0\left(\right.$ ou $\left.\mu_{1}=\mu_{2}\right)$

$\mathrm{H} 1: \mu_{1}-\mu_{2}>0$

sendo $\mu_{1}$ a média populacional para o material relaminado e $\mu_{2}$ a média populacional para o material laminado.

Calculou-se, então, a estatística de teste $\left(T_{0}^{*}\right)$, o grau de liberdade $(v)$ e o Tcrítico $(T)$ :

$$
\begin{gathered}
T_{0}^{*}=\frac{\bar{X}_{1}-\bar{X}_{2}}{\sqrt{\frac{S_{1}^{2}}{n_{1}}+\frac{S_{2}^{2}}{n_{2}}}}=\frac{21,498-21,370}{\sqrt{\frac{0,059^{2}}{30}+\frac{0,098^{2}}{30}}}=6,12 \\
v=\frac{\left(\frac{s_{1}^{2}}{n_{1}}+\frac{s_{2}^{2}}{n_{2}}\right)^{2}}{\frac{\left(\frac{s_{1}^{2}}{n_{1}}\right)^{2}}{n_{1}-1}+\frac{\left(\frac{s_{2}^{2}}{n_{2}}\right)^{2}}{n_{2}-1}}=\frac{\left(\frac{21,498^{2}}{30}+\frac{21,370^{2}}{30}\right)^{2}}{\frac{\left(\frac{0,059^{2}}{30}\right)^{2}}{30-1}+\frac{\left(\frac{0,098^{2}}{30}\right)^{2}}{30-1}}=47
\end{gathered}
$$

Tcrítico $=T_{0,05 ; 47}=1,67$

Pelos cálculos apresentados, nota-se que o $T_{0}^{*}(6,12)$ é maior que o Tcrítico $(1,67)$. Logo, a hipótese H0 foi rejeitada e, consequentemente, a hipótese H1 foi aceita. Sendo assim, pôde-se afirmar com $95 \%$ de confiança que, de fato, a média reduziu, do material relaminado para o material laminado.

Finalmente, foram feitas as análises para constatação das diferenças encontradas no ensaio de arrancamento por tração. Nesse ensaio, para o material relaminado, obteve-se uma força média de $5104,2 \mathrm{~N}$, com desvio padrão de $247,8 \mathrm{~N}$. Para o material laminado, obteve-se uma média de $4850,6 \mathrm{~N}$, com desvio padrão de $261,9 \mathrm{~N}$. Assim como para a análise da variabilidade no processo, fez-se primeiramente um teste F para inferir sobre o desvio padrão com $95 \%$ de confiança, conforme segue:

Teste de hipótese

$\mathrm{H} 0: s_{1}^{2}=s_{2}^{2}$

$\mathrm{H} 1: s_{1}^{2}<s_{2}^{2}$

\section{Valor do Fcrítico}

$F i=F_{29 ; 29 ; 5 \%}=0,53$ 
sendo $s_{1}^{2}$ a variância para o material relaminado e $s_{2}^{2}$ a variância para o material laminado.

Estatística de teste:

$F_{\text {teste }}=\frac{\min \left(247,8^{2} ; 261,9^{2}\right)}{\max \left(247,8^{2} ; 261,9^{2}\right)}=\frac{247,8^{2}}{261,9^{2}}=0,89$

Como o Fteste $(0,89)$ apresentou-se maior que o Fcrítico inferior $(0,53)$, rejeitou-se $\mathrm{H} 1$ e aceitou-se H0. Dessa forma, pôde-se concluir, com 95\% de confiança, que não houve evidências de que o desvio padrão aumentou, ou seja, não houve influência da mudança do material relaminado para o material laminado em relação à variabilidade da força de extração no teste de arrancamento.

Assim, como não há evidências de que as variâncias sejam diferentes, o teste mais adequado utilizado para inferir sobre as médias foi o teste $t$ para diferença de médias de duas populações com distribuições normais presumindo variâncias equivalentes (Montgomery, 2004). Consideraram-se, então, as seguintes hipóteses:

H0: $\mu_{1}-\mu_{2}=0\left(\right.$ ou $\left.\mu_{1}=\mu_{2}\right)$

$\mathrm{H} 1: \mu_{1}-\mu_{2}>0$,

sendo $\mu_{1}$ a média populacional para o material relaminado e $\mu_{2}$ a média populacional para o material laminado.Calculou-se, então, o estimador da variãncia $\left(S_{p}^{2}\right)$ e a estatística de teste $\left(T_{0}\right)$ :

$S_{p}^{2}=\frac{\left(n_{1}-1\right) S_{1}^{2}+\left(n_{2}-1\right) S_{2}^{2}}{n_{1}+n_{2}-2}=\frac{(30-1) 247,8^{2}+(30-1) 261,9^{2}}{30+30-2}=65011,5$

$T_{0}=\frac{\bar{X}_{1}-\bar{X}_{2}}{S_{p} \sqrt{\frac{1}{n_{1}}}+\frac{1}{n_{2}}}=\frac{5104,2-4850,6}{255 \sqrt{\frac{1}{30}+\frac{1}{30}}}=3,85$

Tcrítico $=T_{0,05 ; 58}=1,67$

Assim, como o $\mathrm{T}_{0}$ encontrado $(3,85)$ é maior do que o Tcrítico $(1,67)$, rejeitou-se $\mathrm{H} 0$ e aceitou-se H1. Portanto, pôde-se concluir com $95 \%$ de confiança que, de fato, a força média para a desconexão no teste de arrancamento por tração diminuiu, do material relaminado para o material laminado. Sabe-se, no entanto, que a especificação para este teste é que o produto resista a uma foça mínima de $3800 \mathrm{~N}$. Mesmo ocorrendo a diminuição no valor da força do material relaminado para o material laminado, a nova força média obtida ainda está bem acima do mínimo requerido, considerando-se que o desvio padrão não mudou.

Uma vez analidados os dados e constatado que, apesar de algumas diferenças, o produto fabricado com material laminado continua a atender às especificações dos testes funcionais mais críticos e mantém aceitáveis os padrões de variabilidade de processo, partiu-se para a fase melhorar (I) do DMAIC. Foi, portanto, implementado o material laminado a todos os produtos que usavam o material relaminado em todas as suas variações. Essa implementação envolveu diversos processos de documentação para homologação de produtos nos clientes, 
modificações em desenhos reajustes nos sistemas da empresa, principalmete os que relacionavam-se com os custos dos produtos entre muitas outras atividades as quais demandaram um tempo de aproximadamente seis mêses até que toda a iplementação estivesse completa. Em relação à última fase do DMAIC, controlar (C), foram mantidos os mesmos procedimentos que eram aplicados quando se utilizava o material relaminado.

\section{ANÁLISE DOS RESULTADOS}

$\mathrm{Na}$ análise dos resultados procurou-se mostrar, em maior foco, as comparações financeiras dos resultados da modificação. A primeira análise foi a comparação dos gastos anuais dos dois tipos de material. A diferença entre eles não foi proporcionalmente equilibrada para todas as variações (de P1 a P19). Segundo informações obtidas nas entrevistas, esse desequilíbrio proporcional deve-se aos fatores considerados nas negociações com o fornecedor, como volume de compra, dimensões, níveis de dificuldade para processamento do material, etc. Entretanto, não foram disponibilizadas informações suficientes para que pudesse ser feita uma ponderação mais detalhada em relação a essa comparação. Todavia, com as informações disponibilizadas, pôde-se avaliar a eficiência da aplicação do Seis Sigma de forma simples e conclusiva.

A figura 5 mostra um novo gráfico de Pareto em relação aos gastos anuais com compra de material, agora, laminado. Segundo as informações da empresa, esses valores referem-se exatamente a um mesmo volume de compra em todas as variações. Percebe-se que, diferente da figura 1, dessa vez, as variações de $\mathrm{P} 1$ a P9, com exceção à $\mathrm{P} 7$, representam cerca de $80 \%$ dos gastos com material. Somando-se todos os valores nota-se que houve uma redução nos gastos anuais com compra de material de aproximadamente 18\%, $2 \%$ a menos da meta definida, considerando o valor antigo de $\mathrm{R} \$ 3.716 .104,80$ e o atual de $\mathrm{R} \$ 3.034 .774,60$. Esse novo valor passa a representar aproximadamente $2 \%$ do faturamento anual da empresa, o que permite considerar uma redução de $1 \%$ dessa representatividade em relação ao valor anterior.

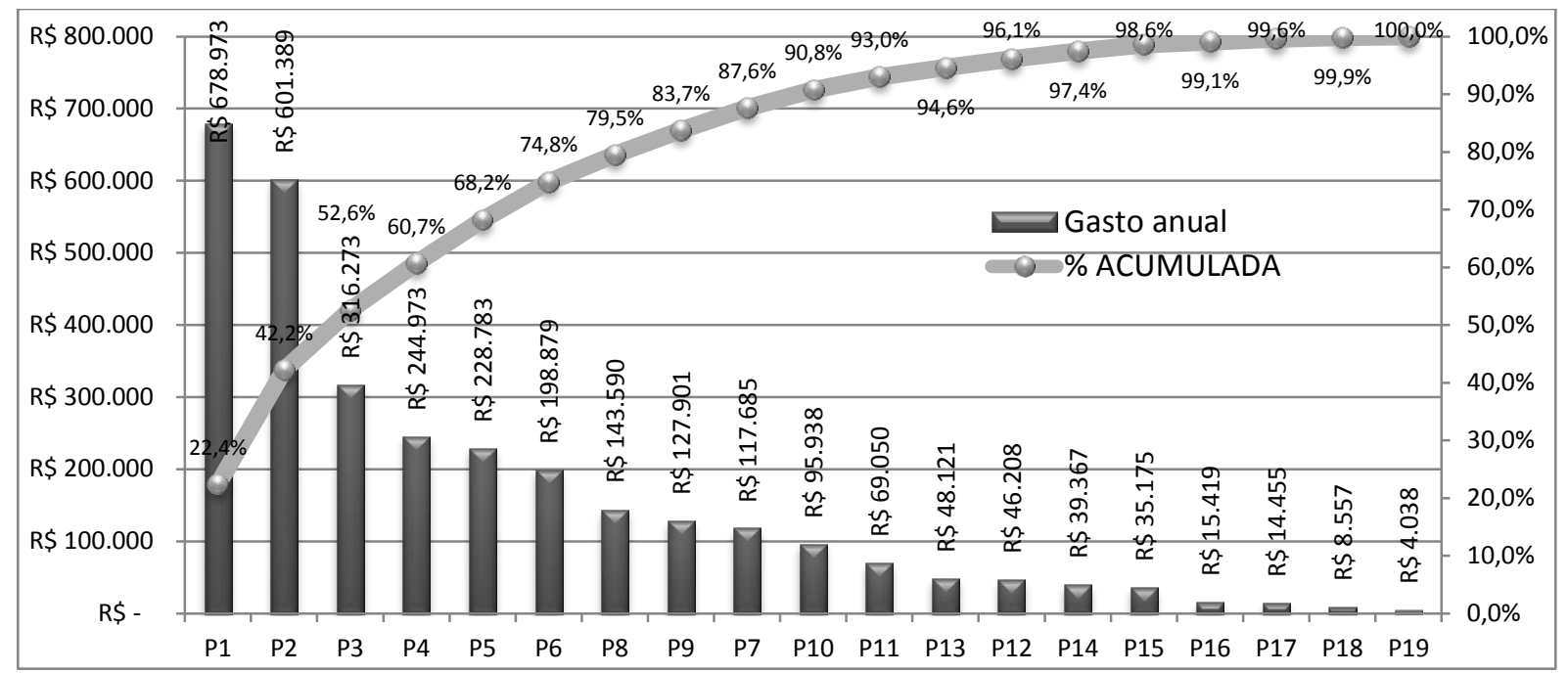

FIGURA 5 - Pareto - Gastos anuais com compra de material laminado 
A figura 6 mostra a diferença dos gastos anuais em relação à mudança do material relaminado para o material laminado. Pelos mesmos motivos supracitados, nota-se que não houve um equilíbrio proporcional nessa diferença. Mesmo assim, a maior redução, em termos de valor monetário, foi a da variação P1, chegando à quantia de R \$224.070, equivalente a 25\%. Já em termos percentuais, a maior redução foi a da variação $\mathrm{P} 12$, com $28 \%$, porém equivalente à uma quantia de apenas $\mathrm{R} \$ 18.323$. Por outro lado, a menor redução, em termos de valor monetário quanto em termos percentuais, foi a da variação P19, com $\mathrm{R} \$ 128$, equivalente a $3 \%$.

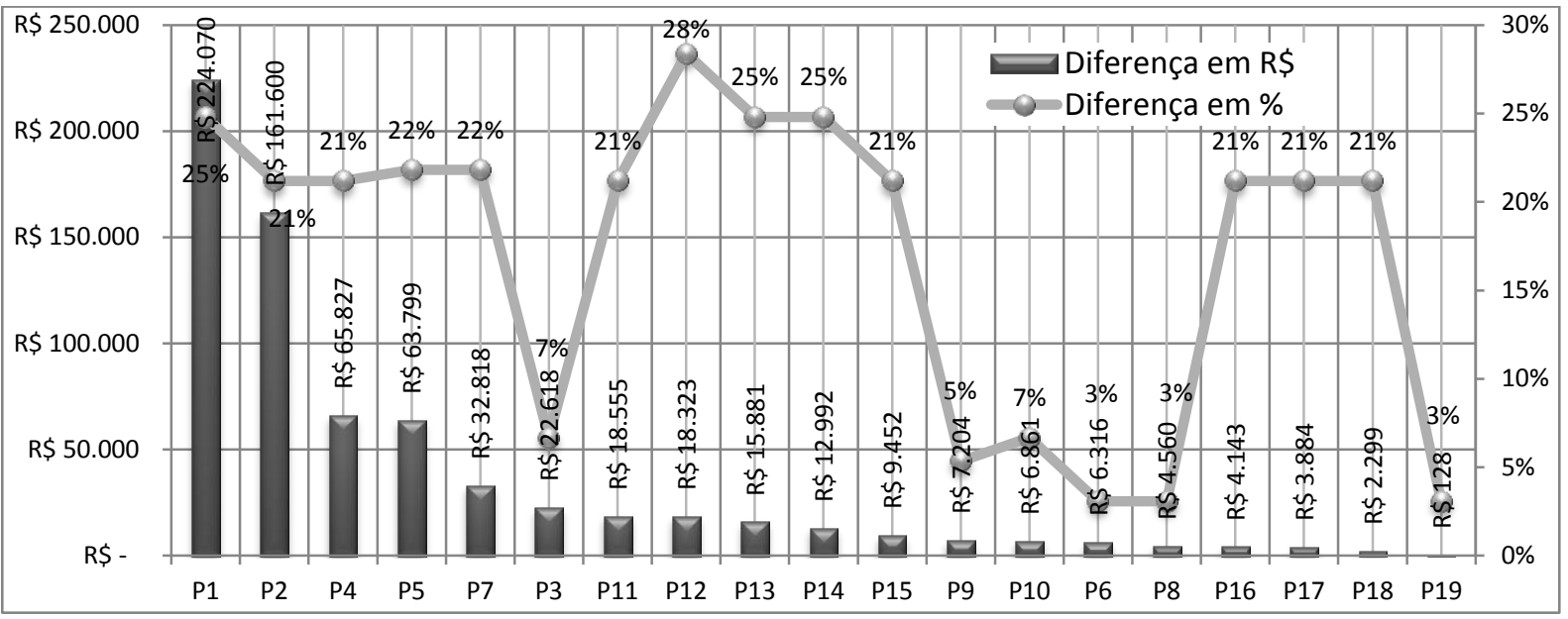

FIGURA 6 - Diferença dos gastos anuais em relação à mudança do material relaminado para o material laminado

Foi realizada outra análise em relação aos resultados que levou em consideração, pelo menos em um dos casos, as diferenças na variabilidade do processo. Para tanto, a empresa forneceu o valor do custo unitário da dobradiça utilizada na análise comparativa do processo com o material relaminado, que é de $\mathrm{R} \$ 0,78$, sendo $\mathrm{R} \$ 0,17$ com a mão-de-obra e $\mathrm{R} \$ 0,61$ com a matéria prima, cuja variação utilizada é a P1. Sabendo-se que os gastos anuais com o material consideram exatamente o mesmo volume em cada variação para os dois materiais, pôde-se estimar um novo custo de matéria prima utilizada na dobradiça com redução percentual igual a dos gastos anuais. Assim, como a redução para P1 foi de $25 \%$, o custo unitário da dobradiça em questão fabricada com o material laminado passa a ser de $\mathrm{R} \$ 0,46,25 \%$ a menos (tabela 2). No custo total, essa diferença representaria uma diferença de $20 \%$, passando-se de uma valor de $\mathrm{R} \$ 0,78$ para uma valor de $\mathrm{R} \$ 0,63$ (tabela 2 ).

Dessa forma, como as análises de variabilidade do processo revelaram, através dos dados extraídos das cartas de controle e dos histogramas (figuras 3 e 4), que o PPM era de 29.912 para o material relaminado e de 7.970 para o material laminado, pôde-se estimar os valores a serem gastos com peças ruins multiplicando-se os valores de PPM pelos valores de custo com matéria prima. Assim, para o material relaminado estima-se que se gastava cerca de $\mathrm{R} \$$ $23.417,96$ e, para o material laminado, $\mathrm{R} \$ 5.020,57$, o que implica uma redução de $79 \%$ (tabela 2). Além disso, considerando uma produção de 1.000 .000 de peças, pôde-se também estimar o custo total unitário efetivo. Esse valor foi encontrado dividindo o valor total gasto para a produção de 1.000 .000 de peças pelo valor de peças boas, 1.000 .000 menos o PPM. Assim, para o material relaminado, chegou-se a um custo total unitário efetivo de $\mathrm{R} \$ 0,81 \mathrm{e}$ para o material laminado, $\mathrm{R} \$ 0,63$, gerando uma redução de $21 \%$ (tabela 2). 
TABELA 2 - Análise econômica dos resultados obtidos

\begin{tabular}{|c|c|c|c|}
\hline & $\begin{array}{c}\text { Dobradiça fabricada com } \\
\text { material relaminado }\end{array}$ & $\begin{array}{c}\text { Dobradiça fabricada com } \\
\text { material laminado }\end{array}$ & Redução em \% \\
\hline Custo total unitário & $\mathrm{R} \$ 0,78$ & $\mathrm{R} \$ 0,63$ & $-20 \%$ \\
\hline Custo com MO & $\mathrm{R} \$ 0,17$ & $\mathrm{R} \$ 0,17$ & $-25 \%$ \\
\hline Custo com MP & $\mathrm{R} \$ 0,61$ & $\mathrm{R} \$ 0,46$ & $-73 \%$ \\
\hline $\mathrm{PPM}$ & 29912 & 7970 & $-79 \%$ \\
\hline $\begin{array}{c}\text { Expectativa de perda em } \\
1.000 .000 \text { de peças produzidas }\end{array}$ & $\mathrm{R} \$ 23.417,96$ & $\mathrm{R} \$ 5.020,57$ & $-20 \%$ \\
\hline $\begin{array}{c}\text { Valor total gasto em 1.000.000 } \\
\text { de peças produzidas }\end{array}$ & $\mathrm{R} \$ \mathbf{7 8 2 . 8 9 5 , 1 2 4}$ & $\mathrm{R} \$ 629.933,944$ & $-21 \%$ \\
\hline $\begin{array}{c}\text { Custo total unitário efetivo } \\
\text { (valor total gasto/(1000000- } \\
\text { PPM)) }\end{array}$ & $\mathrm{R} \$ 0,81$ & $\mathrm{R} \$ 0,63$ & \\
\hline
\end{tabular}

\section{CONCLUSÃO}

Através dos resultados e das análises apresentadas, tornam-se evidentes os benefícios proporcionados pela implementação do Seis Sigma. Percebe-se, entretanto, que para o alcance desses benefícios fez-se necessária um série de atividades devidamente estruturadas, possibilitadas pela utilização do DMAIC. É importante considerar também a relevância das ferramentas estatísticas utilizadas, sem as quais a constatação dos resultados alcançados não seria concretizada. É fato que a meta de redução dos gastos anuais em $20 \%$ não foi alcançada, no entanto, os gastos que totalizavam um valor de $\mathrm{R} \$ 3.716 .104,80$ diminuíram para um valor total de $\mathrm{R} \$$ 3.034.774,60, implicando uma redução de $18 \%$, que foi, sem dúvidas, significativa.

Além disso, em relação ao material, pôde-se concluir que, especificamente no caso analisado, foi possível a substituição do aço relaminado com baixo teor de carbono ABNT NBR 5007 pelo aço laminado a frio ABNT NBR 5915-2. Não obstante as diferenças encontradas, as análises mostraram que o produto de maior criticidade fabricado com o segundo material atendeu aos requisitos considerados mais importantes. Dessa forma, é possível afirmar que, dependendo da aplicação, a utilização de um aço menos nobre pode ser considerada, uma vez que, submetido às análises pertinentes, atenda às devidas especificações. Todavia, mais análises devem ser feitas, em áreas diferentes, para que se possam apurar essas constatações com maiores propriedades.

\section{REFERÊNCIAS}

[1] RAMOS, Alberto Wunderler; RIBEIRO, Celma O.; MIYAKE, Dario Ikuo; NAKANO, Davi; LAURINDO, Fernando José Barbin; HO, Linda Lee; CARVALHO, Marly Monteiro de; BRAZ, Moacyr Albano.; BALESTRASSI, Pedro Paulo; ROTONDARO, Roberto G. Seis Sigma: Estratégia Gerencial para a Melhoria de Processos, Produtos e Serviços. São Paulo: Atlas, 2013. 
[2] MAST, Jeroen de; LOKKERBOL, Joran. An analysis of the Six Sigma DMAIC method from the perspective of problem solving. International Journal of Production Economics, n.139, p.604-614, 2012.

[3] SATOLO, Eduardo Guilherme; ANDRIETTA, João Marcos; MIGUEL, Paulo Augusto Cauchick; CALARGE, Felipe Araújo. Análise da utilização de técnicas e ferramentas no programa Seis Sigma a partir de um levantamento tipo survey. Produção, v. 19, n. 2, p. 400416, 2009.

[4] ASSOCIAÇÃO BRASILEIRA DE NORMAS TÉCNICAS. NBR 5007: Tiras relaminadas de aço de baixo teor de carbono para estampagem: Especificação. Rio de Janeiro, 2013. [5] ASSOCIAÇÃO BRASILEIRA DE NORMAS TÉCNICAS. NBR 5915-2: Chapas e bobinas de aço laminadas a frio parte 2: Aços para estampagem. Rio de Janeiro, 2013. [6] YANG, Kai; EL-HAIK, Basem; Seis Sigma: Um Roteiro para o Desenvolvimento do Produto. São Paulo: Educator, 2008.

[7] SANTOS, Adriana Barbosa; MARTINS, Manoel Fernando. Contribuições do Seis Sigma: estudos de caso em multinacionais. Produção, v. 20, n. 1, p. 42-53, 2010.

[8] SHAFER, Scott M.; MOELLER, Sara B. The effects of Six Sigma on corporate performance: An empirical investigation. Journal of Operations Management, v. 30, n. 7, p. 521-532, 2012.

[9] BAÑUELAS, Ricardo; ANTONY, Jiju. Going from Six Sigma to design for Six Sigma: an exploratory study using analytic hierarchy process. The TQM Magazine, v. 15, n. 5, p. 334-344, 2003.

[10] CHAKRAVORTY, Satya S. Six Sigma programs: An implementation model.

International Journal of Production Economics, n.119, p.1-16, 2009.

[11] CARVALHO, Marly Monteiro de; HO, Linda Lee.; PINTO, Silvia Helena Boarin. Implementação e difusão do programa Seis Sigma no Brasil. Produção, v. 17, n. 3, p. 486501, 2007.

[12] MARCONI, Maria de Andrade; LAKATOS, Eva Maria. Fundamentos de metodologia científica. 7. ed. São Paulo: Atlas, 2010.

[13] YIN, Robert k. Estudo de caso - planejamento e métodos. 4. ed. Porto Alegre: Bookman, 2010.

[14] MONTGOMERY, Douglas C.; RUNGER, George C.; HUBELE, Norma Faris. Estatística aplicada à engenharia. 5. ed. São Paulo: LTC, 2004. 